\title{
Idiopathic Left Ventricular Aneurysm Causing Ventricular Tachycardia with 1:1 Ventriculoatrial Conduction and Intermittent Wenckebach Block
}

\author{
Stelios Paraskevaidis*, George Stavropoulos, Vassilios Vassilikos, Yiannis S. Chatzizisis, \\ Kostas Polymeropoulos, Anthony Ziakas, George Dakos and George E. Parcharidis \\ $1^{\text {st }}$ Cardiology Department, AHEPA University Hospital, Aristotle University Medical School, Thessaloniki, Greece
}

\section{INTRODUCTION}

Left ventricular aneurysms (LVAs) can be congenital or acquired. They develop most frequently after myocardial infarction. Other causes include hypertrophic cardiomyopathy, arrythmogenic right ventricular cardiomyopathy, myocarditis, chest trauma, sarcoidosis or Chagas disease [1,2]. LVAs without identifiable cause are considered as idiopathic. Most of LVAs are asymptomatic and are occasionally identified during routine diagnostic procedures. However, in rare cases LVAs may be associated with life-threatening ventricular tachyarrhythmias (mostly ventricular tachycardia, VT) and sudden cardiac death, even as a first manifestation [3]. In most of the cases VT has right bundle branch block morphology consistent with left ventricular origin. Idiopathic LVAs are anatomically distinguished from congenital diverticula, which are characterized by a narrow communication with the ventricle $[2,4]$.

In this report we describe an interesting and rare case of idiopathic LVA associated with sustained monomorphic VT, ventriculoatrial conduction and intermittent Wenckebach block. Management strategies are discussed and our treatment approach is presented.

\section{Case Report}

A 72-year old woman was admitted to our hospital with palpitations, dizziness and fatigue from a few hours ago. The patient had a history of heart failure (NYHA II) from three years ago and also an episode of paroxysmal atrial flutter two years ago. She was free of angina and had no cardiovascular disease risk factors. The family history was unremarkable with respect to cardiac arrest, unexplained syncope, ventricular tachyarrhythmias, or cardiomyopathy. At the time of presentation to the emergency department the patient was receiving carvedilol, valsartan, and furosemide. The baseline 12-lead ECG revealed a sustained monomorphic VT with RBBB morphology and left axis deviation (Fig. 1A). Since tachycardia was poorly tolerated with systolic blood pressure of $80 \mathrm{mmHg}$ an electrical cardioversion was applied and the rhythm was restored to sinus (Fig. 1B). The ECG,

*Address correspondence to this author at the $1^{\text {st }}$ Cardiology Department, AHEPA University Hospital, Aristotle University Medical School, 1 Stilp. Kyriakidi Street, 54636, Thessaloniki, Greece; Tel: +30-2310-994673; Fax: +30-2310-994837; E-mail: stparask@otenet.gr physical examination and laboratory tests after the cardioversion were unremarkable.

The chest X-ray revealed an increased cardiothoracic index. The transthoracic echocardiogram showed dilated left ventricle with a lateral wall aneurysm, left ventricular ejection fraction of $40 \%$ and mild mitral regurgitation (Fig. 2A). The coronary angiography revealed normal coronary arteries, whereas the left ventriculography further confirmed the lateral wall aneurysm (Fig. 2B). Magnetic resonance imaging further confirmed the presence of the LVA (Fig. 2C).

The patient was started on amiodarone per os and after two weeks an electrophysiology study was performed. The programmed ventricular stimulation in the right ventricular apex with 500/230-240 msec basic drive cycle and two extrastimuli reproducibly induced sustained monomorphic VT of $160 \mathrm{bpm}$ associated with hemodynamic instability. That tachycardia, however, had different characteristics from the one at the emergency department as it was associated with left branch bundle block (LBBB) morphology and normal cardiac axis and was terminated by overdrive pacing (Fig. 1C). The LBBB morphology was consistent with septal origin of the VT. Of note, during the inducible VT a 1:1 ventriculoatrial conduction with intermittent Wenckebach block was recorded (Fig. 1D). A cardioverter defibrillator (ICD) was implanted for the secondary prevention of VT and the patient was discharged 2 days after ICD implantation on treatment with carvedilol, amiodarone and angiotensin converting enzyme inhibitor. During a follow-up period of six months no ventricular arrhythmias occurred.

\section{DISCUSSION}

LVAs are quite rare with a prevalence of $0.76 \%$ in 12,271 consecutive adult patients undergoing cardiac catheterization [5]. These aneurysms can be either congenital or acquired. Congenital LVAs are rare, potentially lethal and they represent a developmental anomaly of left ventricular endocardium and myocardium [2]. Acquired LVAs have either cardiac or non-cardiac aetiology. The most frequent cardiac cause of LVAs is myocardial infarction or coronary artery fistula but they can also occur in hypertrophic cardiomyopathy, myocarditis, arrhythmogenic right ventricular cardiomyopathy, blunt chest trauma and surgery. Noncardiac causes of LVAs include Chagas disease, sarcoidosis, lupus erythematosus, rheumatic heart disease, tuberculosis, human immunodeficiency virus, glycogen storage diseases 
A. VT on admission

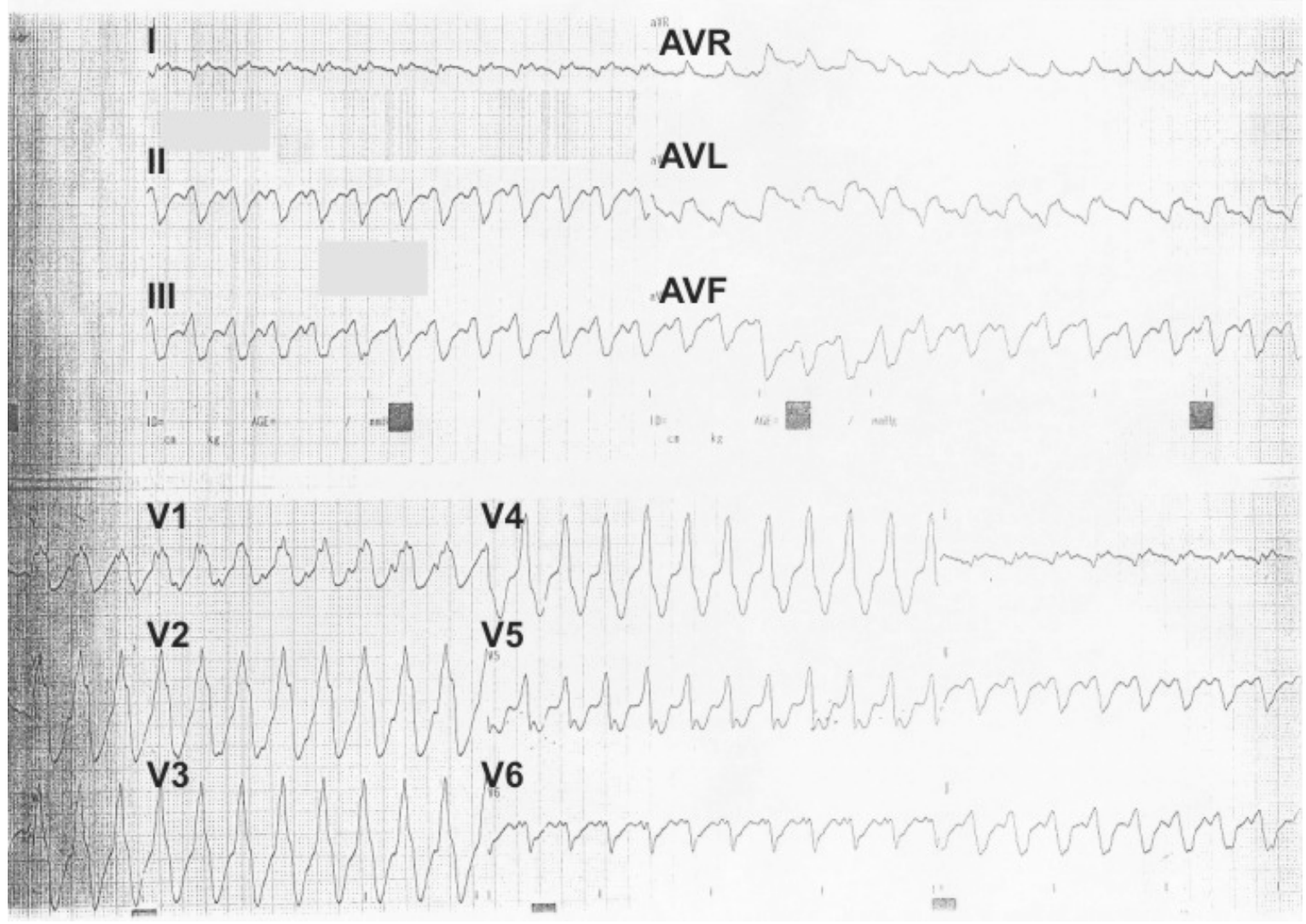

\section{B. Sinus rhythm after cardioversion}
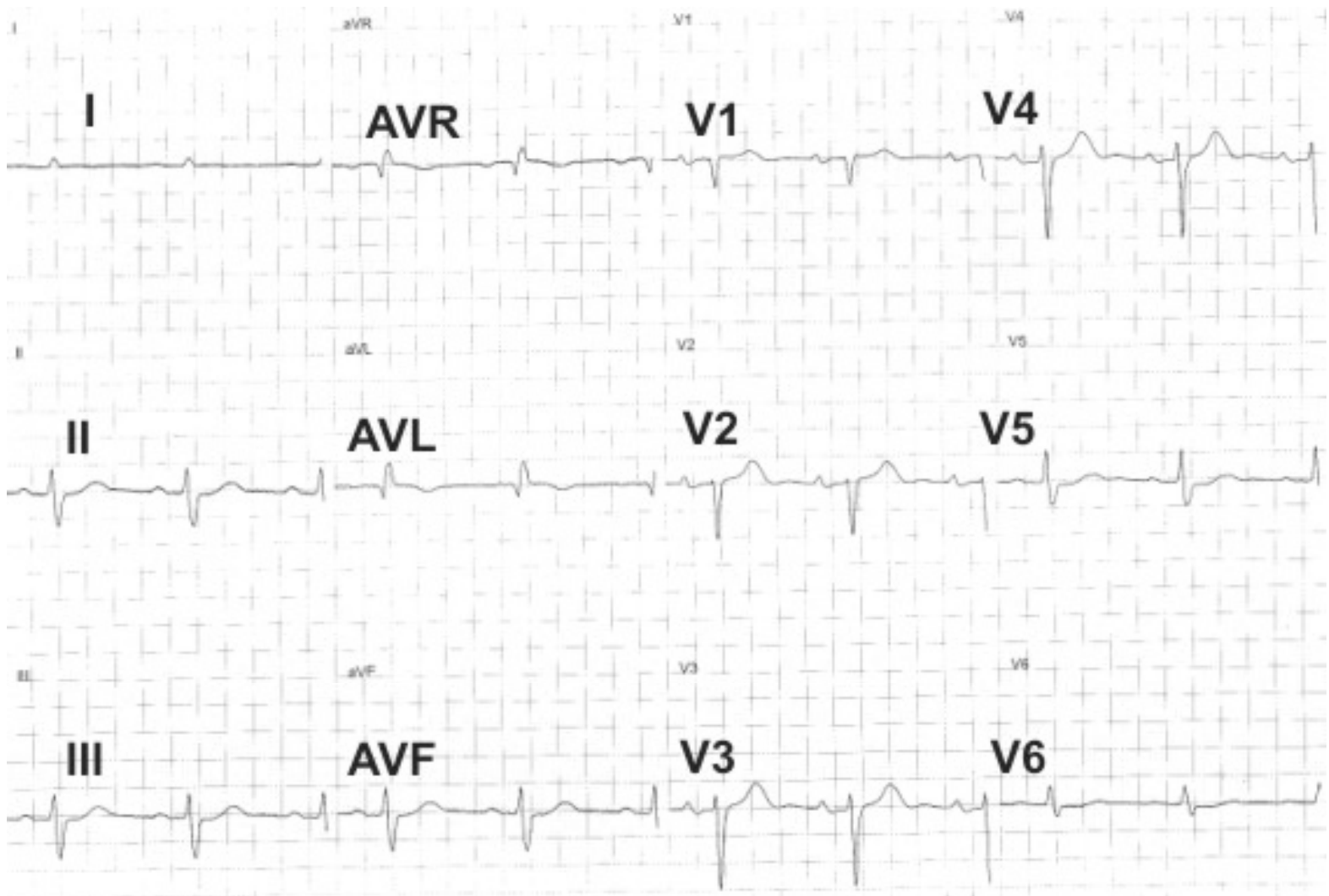


\section{Induced VT}

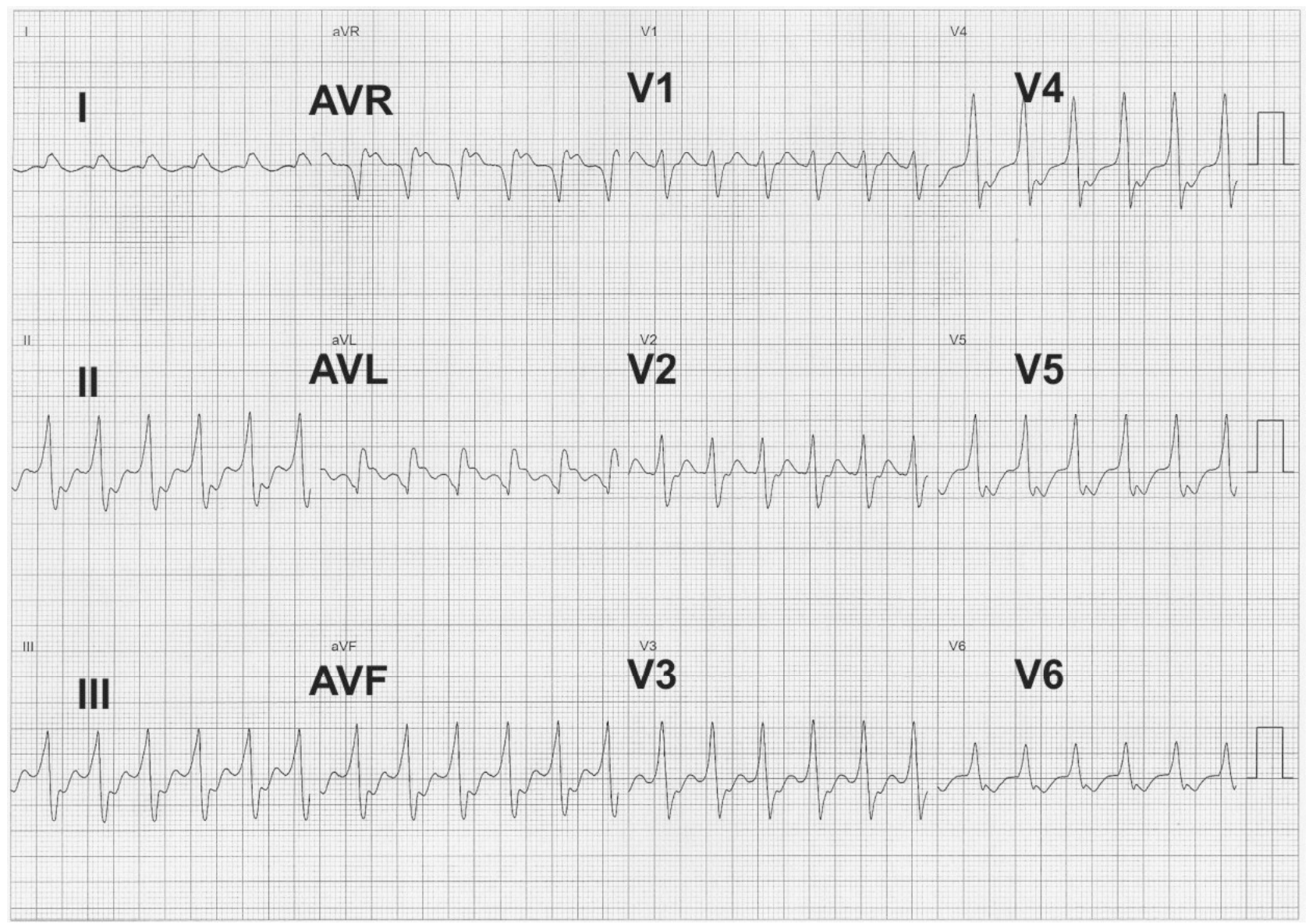

\section{Electrophysiology}

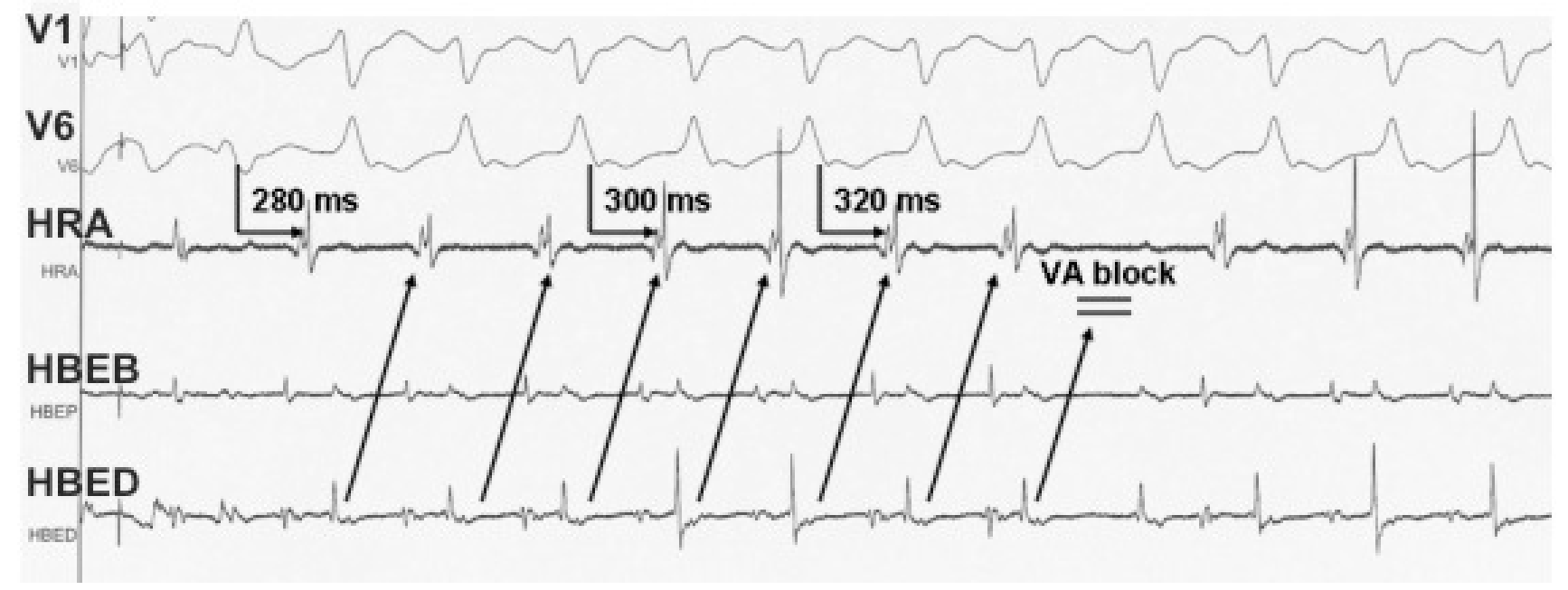

Fig. (1A). ECG on admission showing the ventriculat tachycardia (VT) with RBBB morphology, (B). Restoration of VT to sinus rhythm with electrical cardioversion, (C). Induced VT with LBBB morphology during the electrophysiology study, (D). Electrogram during the electrophysiology study showing a 1:1 ventriculoatrial conduction with intermittent Wenckebach block.

and hyperimmunoglobulin E syndrome [1,3]. LVAs without identifiable cause are considered as idiopathic as in the present case which is quite rare. Patients with LVAs may be either asymptomatic or manifest with recurrent arterial emboli, angina, congestive heart failure, ventricular tachyarrhythmias and sudden cardiac death. As a result, LVAs represent an uncommon but important cause of sudden death, especially in young adults. 

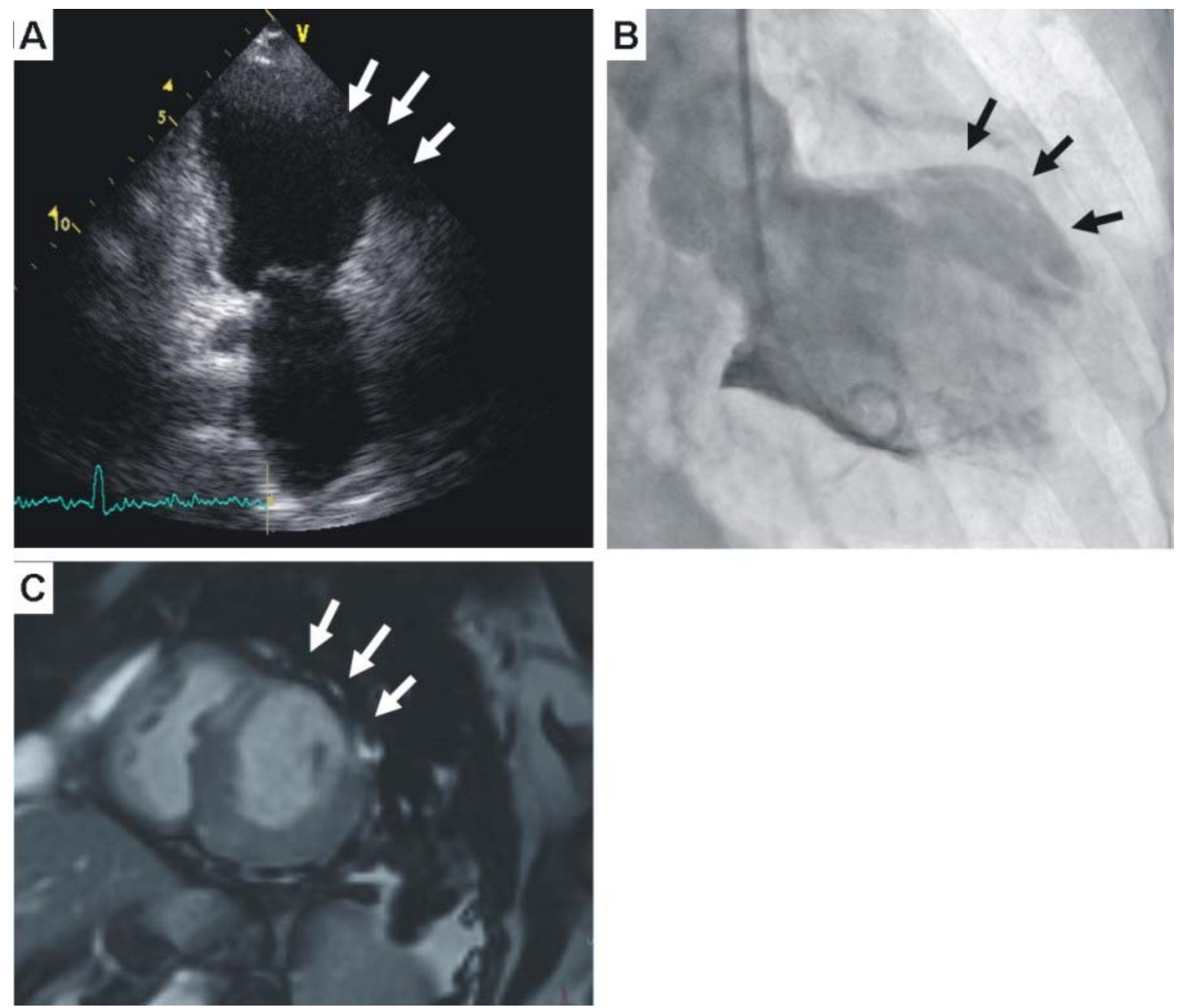

Fig. (2A). Echocardiogram showing the left ventricular aneurysm (arrows), (B). Left ventriculography further depicting the lateral wall aneurysm (arrows), (C). Magnetic resonance imaging further confirmed the presence of an aneurysm with wall thinning (arrows).

In most of the cases, VT associated with LVAs present with RBBB morphology due to the left ventricular origin. In our case this was evident in the clinical tachycardia, but not in the inducible one during the electrophysiology study which had LBBB morphology. The LBBB morphology was indicative of a septal origin of the tachycardia and potentially indicated global myocardial involvement beyond the lateral wall where the aneurysm was located. Of note, during the induced VT a 1:1 ventriculoatrial conduction with intermittent Wenckebach block was revealed which is a quite unusual finding. This block enabled us to differentiate the VT from supraventricular tachycardias with LBBB aberration and 1:1 ventriculoatrial conduction, such as atypical atrioventricular node reentry tachycardia and atrioventricular reentry tachycardia due to septal accesory pathways.

Treatment of LVAs depends on the presence of symptoms of heart failure, arterial emboli and ventricular tachyarrhythmias. The ventricular tachyarrhythmias can be treated by antiarrhythmic drugs, VT ablation, or by an ICD implantation as in our case [6]. Aneurysmectomy can also be an option especially if conservative approaches are not effective [7]. However, prospective long-term follow-up studies comparing the different management strategies are missing.
Prognosis of idiopathic LVAs depends on their size, location, degree of valvular involvement, functional class of congestive heart failure and the presence of ventricular tachyarrhythmias [1, 8]. In our case, the implantation of ICD complemented with amiodarone per os was a quite effective treatment strategy, as no arrhythmia was reported in the following six months.

In conclusion, we present a case with idiopathic LVA presented with VT, 1:1 ventriculoatrial conduction and intermittent Wenckebach block. The patient was successfully treated with an ICD and remained asymptomatic in the subsequent six months.

\section{REFERENCES}

[1] Paul M, Schäfers M, Grude M, et al. Idiopathic left ventricular aneurysm and sudden cardiac death in young adults. Europace 2006; 8: 607-12.

[2] Papagiannis J, Van Praagh R, Schwint O, et al. Congenital left ventricular aneurysm: clinical, imaging, pathologic, and surgical findings in seven new cases. Am Heart J 2001; 141: 491-9.

[3] Tada H, Kurita T, Ohe T, et al. Clinical and electrophysiologic features of idiopathic left ventricular aneurysm with sustained ventricular tachycardia. Int J Cardiol 1998; 67: 27-38.

[4] Giannoglou GD, Delioglanis SH, Paraskevaidis S, et al. Ventricular diverticulum: definition, pathophysiology, clinical manifestation and treatment. Aristotle University Med J 2007; 34: 87-92. 
[5] Ohlow MA, Secknus MA, Geller JC, et al. Prevalence and outcome of congenital left ventricular aneurysms and diverticula in an adult population. Cardiology 2008; 112: 287-93

[6] Santamaria M, Cireddu M, Riva S, et al. Radiofrequency catheter ablation guided by noncontact mapping of ventricular tachycardia originating from an idiopathic left ventricular aneurysm. J Interv Card Electrophysiol 2007; 19: 49-53
[7] Tsai CL, Chang WT, Chen WJ. Idiopathic left ventricular aneurysm. Eur J Cardiothorac Surg 2004; 26: 438-9.

[8] Frustaci A, Chimenti C, Pieroni M. Prognostic significance of left ventricular aneurysm with normal global function caused by myocarditis. Chest 2000; 118: 1696-702.

Received: July 17, 2009

Revised: July 24, 2009

Accepted: July 27, 2009

(C) Paraskevaidis et al.; Licensee Bentham Open.

This is an open access article licensed under the terms of the Creative Commons Attribution Non-Commercial License

(http://creativecommons.org/licenses/by-nc/3.0/) which permits unrestricted, non-commercial use, distribution and reproduction in any medium, provided the work is properly cited. 